\title{
Effect of scanner acoustic background noise on strict resting-state fMRI
}

\author{
C. Rondinoni ${ }^{1}$, E. Amaro Jr. ${ }^{2}$, F. Cendes $^{3}$, A.C. dos Santos ${ }^{1}$ and C.E.G. Salmon ${ }^{4}$ \\ ${ }^{1}$ Departamento de Clínica Médica, Faculdade de Medicina de Ribeirão Preto, Ribeirão Preto, SP, Brasil \\ ${ }^{2}$ Departamento de Radiologia, Faculdade de Medicina, Universidade de São Paulo, São Paulo, SP, Brasil \\ ${ }^{3}$ Departamento de Neurologia, Faculdade de Medicina, Universidade Estadual de Campinas, Campinas, SP, Brasil \\ ${ }^{4}$ Departamento de Física, Faculdade de Filosofia, Ciências e Letras de Ribeirão Preto, Universidade de São Paulo, \\ Ribeirão Preto, SP, Brasil
}

\begin{abstract}
Functional MRI (fMRI) resting-state experiments are aimed at identifying brain networks that support basal brain function. Although most investigators consider a 'resting-state' fMRI experiment with no specific external stimulation, subjects are unavoidably under heavy acoustic noise produced by the equipment. In the present study, we evaluated the influence of auditory input on the resting-state networks (RSNs). Twenty-two healthy subjects were scanned using two similar echo-planar imaging sequences in the same 3T MRI scanner: a default pulse sequence and a reduced "silent" pulse sequence. Experimental sessions consisted of two consecutive 7-min runs with noise conditions (default or silent) counterbalanced across subjects. A selforganizing group independent component analysis was applied to fMRI data in order to recognize the RSNs. The insula, left middle frontal gyrus and right precentral and left inferior parietal lobules showed significant differences in the voxel-wise comparison between RSNs depending on noise condition. In the presence of low-level noise, these areas Granger-cause oscillations in RSNs with cognitive implications (dorsal attention and entorhinal), while during high noise acquisition, these connectivities are reduced or inverted. Applying low noise MR acquisitions in research may allow the detection of subtle differences of the RSNs, with implications in experimental planning for resting-state studies, data analysis, and ergonomic factors.
\end{abstract}

Key words: Resting-state fMRI; Acoustic noise; Default-mode network; Independent component analysis; Granger causality mapping

\section{Introduction}

The wax-and-waning mind process that lingers during a resting-state functional session is thought to be mediated by a set of brain areas known as resting-state networks (RSNs), which mediate the interplay between salience detection and execution triggering $(1,2)$. The most ubiquitous among these networks has been named Default-mode Network (DMN), consisting of brain areas that are "deactivated" when voluntary action is required. This finding led to the notion that an organized mode of brain function takes place and defines a baseline, which is partially suspended during directed action (3).

The extent to which the brain is engaged or disengaged in a task during a functional exam has been a matter of debate in the literature (3). The inherent acoustic noise of the magnetic resonance imaging (MRI) scanner can bias brain activity towards action voluntarily inhibiting the uncomfortable high noise, in contrast to a resting condition. The main sources of acoustic noise during an
MRI experiment are the gradient coil mechanical vibrations, coolant air conditioning, and the ventilation apparatus. Previous studies have indicated that the consequence of the stray noise regarding blood oxygenation level-dependent (BOLD) dynamics may be understood as effects of saturation or masking (4-9). This is especially true for the working memory function, as shown by higher recruitment of attentive resources in the presence of loud acoustic noise. BOLD activation was found to be higher in the cerebellum, frontal cortex, fusiform cortex, and lingual gyrus and lower in the anterior cingulate and putamen when a standard functional acquisition was compared to sparse acquisition volumes acquired with interspaced periods of silence (10). It has been suggested that, by arresting attentive resources, the noise could non-linearly modulate a set of intrinsic networks, especially when tasks are designed to study auditory perception (11). A recent study used the same

Correspondence: C.E.G. Salmon, Departamento de Física, Faculdade de Filosofia, Ciências e Letras de Ribeirão Preto, USP, Av. Bandeirantes, 3900, 14040-901 Ribeirão Preto, SP, Brasil. Fax: +55-16-3602-4887. E-mail: garrido@ffclrp.usp.br 
approach to investigate the influence of stray noise on RSNs, showing the robustness of the RSNs (12). These authors also recognized that, although the comparison between sparse and continuous acquisitions is legitimate, unavoidable criticisms arise due to the intermittent noise that accompanies sparsely acquired scans, retaining an alarming effect and spoiling the tenets of a strict restingstate paradigm.

Three main approaches are proposed to overcome the possible influences or confounds introduced in brain function by high noise levels. First, the interaction between software and hardware offers the possibility of attenuating sound and reducing the sound levels by decreasing the slew rate and rise time of gradient pulses (13), by applying long sine waves (14), and by using active noise control with adaptive wave cancellation algorithms (15). Second, investigators can physically attenuate the noise by using earmuffs or earplugs in order to impose physical barriers against the audible parts of the noise spectrum (16-19). Yet, the acoustic conduction by bone and soft parts of the head may hinder the full success of this approach. Finally, the experimental paradigm may be modified into a sparsely acquired echo-planar imaging (EPI) sequence, when the acquisitions of functional volumes are interspaced with the silent periods, in such a way as to avoid BOLD responses related to the noise itself $(5,20,21)$. These approaches to noise reduction have several practical limitations, as noted elsewhere $(6,7)$. Therefore, efforts are constantly renewed by scientific associations dedicated to regulating the field of research and clinical practice by offering periodic reviews of the guidelines for the safety of volunteers, patients, and technical workers $(22,23)$.

Besides environmental factors, an issue frequently raised in the analysis of resting data is the statistical approach to be applied. Besides BOLD signal correlation between a seed volume-of-interest (VOI) and remaining whole brain voxels, a common way of analyzing data for intrinsic patterns without assuming any prior assumptions is the independent component analysis (ICA) $(24,25)$. The algorithm is based on an adaptive filter that maximizes the independence of the temporal series components by decreasing the mutual information. The ICA algorithm is applied to whole-brain voxels in order to separate the set of information into networks with maximally independent fluctuations. The resulting individual ICA maps reveal two complementary principles underlying the study of brain organization: localization and connectionism (26). While the localization approach considers that a given brain function is accomplished by a finite set of specific and segregated brain areas, connectionist views consider that these areas may be widely distributed and functionally connected across space. This permits group activity maps to be consistently achieved by applying a clustering method to individual ICA maps of different subjects, indicating areas of common activity across individuals (27).
As reviewed by Friston (28), one should note that maps gathered by correlation or independent analysis reflect statistical dependencies that bear confounds related to inputs from other brain areas, although indicating functional connectivity. Algorithms based on direct causality or temporal auto-regression offer a way to reduce such confounds, yielding maps of effective connectivity based upon a priori models of connectivity. As in Granger causality mapping (GCM) algorithms, effective connectivity is calculated by quantifying the usefulness of taking the value of a given voxel time series to predict the values of another voxel; that is, one voxe will Granger-cause another if past values of the former improve the prediction of the current values of the latter (29). Although the hemodynamic blurring of the MRI signal and the relatively low temporal resolution of the acquisition may be considered drawbacks in applying Granger causality models to the context of fMRI, the results of simulations indicate that the approach is feasible and yields valid results. Difference in GCM values may indicate the direction of the causality since the signal from the calculation reference to voxels minus voxels to reference shows if the BOLD in the reference is Granger-causing or being Granger-caused by the other voxels entering the analysis (29).

Here, we used the resting-state functional connectivity approach to determine if the massive input of auditory information could mask or alter the brain networks. Previous research by our group indicates that there are differences in the levels of functional connectivity under different levels of acoustic noise (30). Should that be confirmed, we would investigate the consequences of higher noise levels on effective connectivity across brain areas. Results are discussed in terms of their implications for the experimental design, data quality, and ergonomic factors in the MRI environment.

\section{Subjects and Methods}

\section{Subjects and acquisition procedures}

Twenty-two right-handed volunteers were recruited for the study (11 females, group means \pm SD age: $29 \pm 8$ years) from the academic community on the Ribeirão Preto Campus of the University of São Paulo. Inclusion criteria were no neurological or psychiatric disorder, no ingestion of psychoactive medications prior to participating in the study, and normal hearing reported by the subjects. The research protocol was approved by the local Internal Review Board (Comitê de Ética em Pesquisa do HCRP e FMRP-USP \#1335/2011) and all subjects gave written informed consent. Data were collected between June 2011 and February 2012. Subjects were instructed to remain still with eyes open and fixating through the head coil mirror. Functional images were acquired in two runs of $7 \mathrm{~min}$ each, after which subjects could close their eyes for the remaining structural acquisitions. Head 
movements were avoided by the use of cushions between the earmuffs and the head coil. Subjects were instructed to relax their neck and posterior part of the trunk, as a means of avoiding excessive movement due to accommodation during image acquisition.

\section{Noise measurements}

Noise differences were assessed by recording the EPI gradient sounds with a microphone inside the scanner room during standard and silent acquisitions. Noise recording and analysis were done using the Audacity software (audacity.sourceforge.net) with a sampling frequency of $44.1 \mathrm{kHz}$. The power spectrum of recorded soundtracks was generated by fast Fourier transform using a 256-data point Hann window.

\section{Acquisition parameters}

The images were acquired with a Philips Achieva 3T MRI equipment (Best, The Netherlands) using a standard 8-channel head coil. Both echoplanar sequences had the following parameters: 200 volumes, 29 slices in ascending order without gaps, 4-mm slice thickness, voxel sizen $=3 \times 3 \mathrm{~mm}$, field of view $=240 \times 240 \mathrm{~mm}$, TR/TE $=$ $2000 / 30 \mathrm{~ms}$. The silent sequence was designed by setting to maximal (level 5) the "soft-tone" parameter offered by the MRI equipment, which decreases the gradient slew rate, leading to lower coil vibration levels. During standard noise conditions, the "soft-tone" parameter was turned off while all other image parameters were kept constant. Each subject was scanned for the acquisition of anatomical 3-D T1-weighted images (TR $=9.7 \mathrm{~ms}$; TE $=4 \mathrm{~ms}$; flip angle $=12^{\circ}$; matrix $=256 \times 256 ; \mathrm{FOV}=256 \mathrm{~mm}$; voxel size $=1 \times 1 \times 1 \mathrm{~mm}$ ).

\section{Functional data analysis}

Data were processed with the BrainVoyager software (Brain Innovations, The Netherlands) (31). Functional volumes were corrected for 3-D motion with reference to the first volume. Subjects who showed movements larger than $2 \mathrm{~mm}$ were excluded. Slice order correction and coregistration to the anatomical volume were done before standardization into the Talairach space. After linear drift filtering, functional data entered the ICA algorithm, which resulted in individual ICA maps thresholded for clusters larger than 150 voxels and z-ICA values higher than 3.5. BOLD components were identified depending on the fingerprint of each individual ICA map (32), leading to a classification of brain networks as proposed elsewhere (27). Components showing high spectral densities between 0.02 and $0.05 \mathrm{~Hz}$, high values of clustering and skewness, and low kurtosis were selected and considered a component related to the canonical BOLD signal.

Group ICA maps were calculated for each noise condition by a self-organizing clustering algorithm in BrainVoyager considering random effects (31). In this assumption, each individual subject of a study is a representative sample of a population. We classified the group ICA maps into representative functional RSNs (Default-mode, Dorsal Attention, Visual, Motor/Auditory, Executive, Extrastriate Visual, Supplementary Motor, Hippocampal, and Entorhinal Networks) based on the comparison with previously published data (27). Group components were selected from the most consistent functional RSNs for further processing. These components were thresholded $(z-I C A>2.0)$ to define volume masks, referred to here as RSN templates.

Voxel-wise maps were created by entering the group components (DMN, Visual, Motor/Auditory, Executive, and Dorsal Attention Network) under each acoustic noise condition into a voxel-wise two-sample two-tailed $t$-test. Brain areas with significant differences were identified in the Talairach space. To confirm that the effects shown by our data depended on the stray noise and not on any combined effects of acoustic noise and subject exhaustion, we compared group ICA maps from the first and the second acquisition runs.

In the second step of the analysis, those brain regions that showed differences in the comparison between RSNs under standard and silent EPI sequences were used as seed-regions for GCM (29). The functional volumes were submitted to spatial smoothing of $8-\mathrm{mm}$ full-width at half maximum, linear trend removal, and temporal high-pass filtering with 2 cycles during the whole run. Spatial smoothing was applied to adjust the individual data to the Brodmann template, permitting the comparison of maps across subjects. The linear trend removal step was required to use the model that relied on vector autoregressive models since the calculations claimed a stationary time series for a consistent computation. Figure 1 summarizes the steps of the GCM analysis, namely, 1) seed VOls were defined in brain regions with voxel-wise ICA differences; 2) Granger causality values between these seeds and the whole brain were calculated; 3) Granger maps were overlapped with RSN templates, resulting in Granger causality values in each condition for the several RSN templates; 4) the paired $t$ test indicated which network showed Granger causality difference (to/from each seed) between noise conditions and across subjects.

\section{Results}

The power spectra of the sound pressure in each scanner setting are shown in Figure 2. The mean difference in Fourier sound spectrum was $12 \mathrm{~dB}$ (standard tone: $68 \mathrm{~dB}$, silent tone: $56 \mathrm{~dB}$ ), which is in agreement with previous studies modifying the frequency of the EPI-readout train $(11,33)$.

Table 1 lists the RSNs found under both conditions. Along with the canonical RSN, our results showed further spontaneous activity in networks not previously described, i.e., supplementary motor and hippocampal 


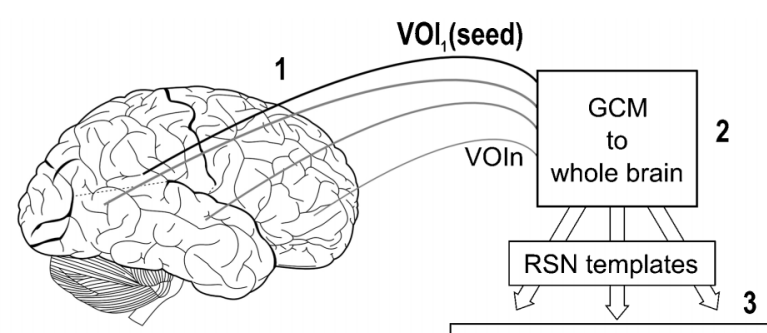

Granger causality values

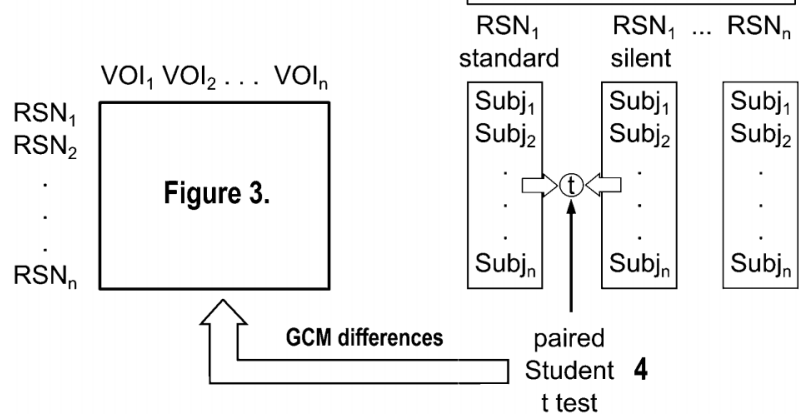

Figure 1. General scheme showing the approach of our data analysis. Numbers stand for $1=$ seed definition based on between-group ICA differences; 2 = Granger causality values calculation across whole brain voxels; $3=$ Granger maps and RSN templates overlapped; $4=$ paired $t$-test for GCM differences between noise conditions and across subjects (see Figure 3). VOI = volume-of-interest; Subj = subject; RSNs = resting-state networks; GCM $=$ Granger causality mapping.

networks. Also, motor and auditory networks were found in the same independent components. The random-effect group ICA at the two different noise levels indicated the existence of 7 common functional networks. The Entorhinal Network was exclusive for standard acquisition, while the Extrastriate Visual Network was found only under silent EPI.

A paired $t$-test comparison was made between the 5 main networks (Default-mode, Visual, Dorsal Attention

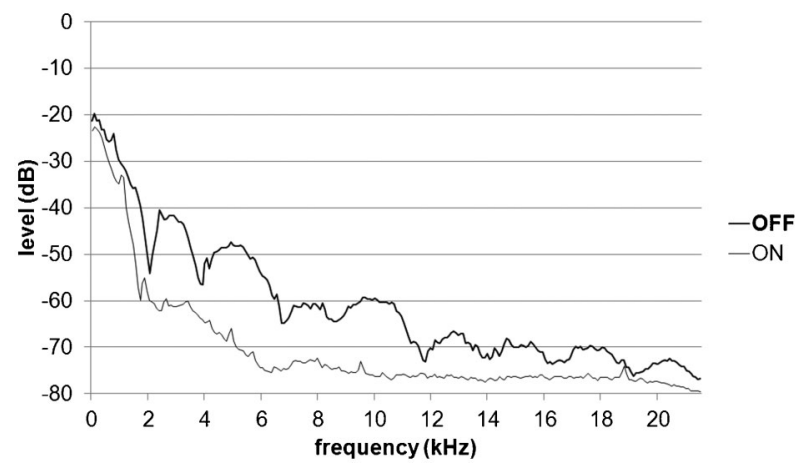

Figure 2. Sound spectra produced by a standard MRI sequence [soft-tone (OFF)] and silent sequence [soft-tone (ON) level 5]. Mean attenuation was $12 \mathrm{~dB}$ in the comparison between standard and silent spectra (range: $86-21963 \mathrm{~Hz}$ ).
Network, Auditory-Motor, and Executive) under each noise condition. Table 2 lists the comparison results that yielded regions of interest. The difference map obtained from the comparison between the first and the second runs did not show significant clusters of activity, as defined by z-scores (z-ICA) higher than 3.5 and clusters larger than 350 voxels.

The GCM algorithm requires seed VOls with about 300 voxels. The resulting VOI in the right insula had 1400 voxels and reached 3 different Brodmann areas (BA; 13, 22 , and 38 ). Thus, 3 smaller volumes were located in the insula, equally spaced along the dorsoventral axis and located in different BA (Table 2).

Figure 3 depicts the mean values for differential Granger causality from seed regions to RSN templates. Significant differences in GCM values were found when a seed was placed in the right inferior insula (BA 38), left middle frontal, right precentral, and left inferior parietal lobule. Differences in GCM values were considered to be significant at $\mathrm{P}<0.05$.

\section{Discussion}

The motivation of this research is the concern about the influences of environmental noise on the organized brain function at rest. Over the last few years, restingstate paradigms have become the approach of choice for many investigators, given their simplicity and robustness and their applicability to a wide variety of subjects. In this way, the concern about the influence of the acoustic noise on the RSNs stems from the fact that a whole body of research could be looking at no rest at all, but into networks that are, in essence, defending the sensory system from an annoying massive input of acoustic noise.

The noise measurements showed that the reduction of sound pressure levels (SPL) reached the same amplitudes as previously described (11). The mean reduction of SPL across the spectrum was $12 \mathrm{~dB}$ from standard to silent sequences, which was found when using slew rate reduction as a strategy for lower SPL during functional exams (13).

The effect of scanner background noise (SBN) may then be "aliased" in the functional data (10), avoiding the identification of the real RSNs. Previous studies scrutinized for the active brain areas by applying the "near-rest" approach $(9,10,12)$, with periods of silent rest intertwined with scanner noise. Near-rest approaches often require that the functional time courses be cropped, giving rise to criticism in analyzing "synthetic" functional data, that would not represent a true and continuous resting condition. The present study attempts for the first time to investigate the brain dynamics under natural and strict resting conditions.

Continuous sampling showed that brain activity was different from that obtained by sparse sampling when EPI volumes were acquired after periods of silence. The SBN seems to have suppressed the default network 
Table 1. Networks detected under both noise conditions, voxel number and mean RFX group z-ICA transformed values inside each network in the presence of each acoustic noise level.

\begin{tabular}{|c|c|c|c|c|}
\hline \multirow[t]{2}{*}{ Putative name } & \multicolumn{2}{|c|}{ Standard EPI } & \multicolumn{2}{|c|}{ Silent EPI } \\
\hline & Voxels & z-ICA value & Voxels & $z-I C A$ value \\
\hline Default-mode network & 33975 & 4.39 & 25688 & 4.56 \\
\hline Dorsal attention network & 13467 & 4.04 & 7654 & 4.13 \\
\hline Visual network & 20647 & 4.15 & 28139 & 4.24 \\
\hline Motor/auditory network & 685 & 3.89 & 30793 & 4.29 \\
\hline Executive network & 3250 & 4.11 & 7679 & 4.36 \\
\hline Extrastriate visual network & - & - & 3593 & 4.14 \\
\hline Supplementary motor network & 1524 & 3.79 & 10789 & 4.10 \\
\hline Hippocampal network & 7914 & 4.43 & 1875 & 4.31 \\
\hline Entorhinal network & 6909 & 3.89 & - & - \\
\hline Total & 88371 & & 116210 & \\
\hline
\end{tabular}

Maps were thresholded with z-ICA values higher than 3.5 and clusters larger than 350 voxels. ICA = independent component analysis; $\mathrm{EPI}=$ echo-planar imaging; RFX = random-effects analysis.

components, like the medial prefrontal cortex, posterior cingulate and precuneus. Gaab et al. (10) reported that the noise does not alter the spatial distribution of the default network, but influences its magnitudes in a nonlinear fashion. This is also true for the working memory functioning (11), as shown by the higher recruitment of attentive resources under high noise conditions. BOLD activation was higher in the cerebellum, frontal cortex, fusiform cortex, and lingual gyrus and lower in the anterior cingulate and putamen. As found in another study assessing SBN (9), the higher attentive demand needed to hear sentences under high acoustic noise resulted in higher activation of the left temporal and inferior parietal cortices. They also concluded that silent EPI sequences would be more adequate for auditory perception studies and their applicability depends on the regions of interest in the brain.
Our global results (Table 1) agree with those cited above. Under lower noise conditions, the networks reach wider areas, leaving room for higher oscillatory activity in the motor/auditory cortex, supplementary motor network, and inside the frontal executive network. On the contrary, during standard EPI acquisition, the aversive nature of the background acoustic noise "dampens" the sensory processes and induces a reduction in the activity of brain areas related to sensorimotor processing. In accordance with a wider oscillation, another interesting aspect of our results is the exclusive network that appears depending on the noise condition. An extrastriate component under silent acquisition points to a more dynamic functioning in associative areas of the brain during lower noise, while a network in the entorhinal cortex may point to an inhibitory activity from this area to the hippocampus under standard EPI (34). In fact, we also found an oscillatory component

Table 2. Brain areas exhibiting differences in the pairwise comparison between functional maps of the 5 main resting-state networks (self-organizing group independent components) under standard and silent acquisitions (two-tailed Student $t$-test).

\begin{tabular}{|c|c|c|c|c|c|c|c|}
\hline \multirow[t]{2}{*}{ Brodmann area } & \multirow[t]{2}{*}{ Topographic region } & \multirow[t]{2}{*}{ Hemisphere } & \multirow[t]{2}{*}{ Voxels } & \multirow[t]{2}{*}{$t$-value } & \multicolumn{3}{|c|}{ Talairach coordinates } \\
\hline & & & & & $x$ & y & z \\
\hline \multicolumn{8}{|c|}{ Higher $z$-ICA for soft-tone acquisition $(P<0.01)$} \\
\hline 40 & Inferior parietal lobule & Left & 528 & 3.14 & -57 & -37 & 28 \\
\hline 40 & Inferior parietal lobule & Right & 1163 & 3.87 & 56 & -34 & 29 \\
\hline 13 & Insula & Right & 1403 & 3.03 & 42 & -5 & 11 \\
\hline 22 & & & & & 50 & -4 & 1 \\
\hline 38 & & & & & 42 & -6 & -8 \\
\hline 9 & Middle frontal gyrus & Left & 473 & 3.56 & -38 & 30 & 30 \\
\hline 6 & Precentral gyrus & Right & 448 & 3.43 & 13 & -14 & 65 \\
\hline \multicolumn{8}{|c|}{ Higher $z-I C A$ for standard tone acquisition $(P<0.01)$} \\
\hline 30 & Posterior cingulate & - & 1168 & 3.78 & -2 & -49 & 2 \\
\hline
\end{tabular}

$\mathrm{ICA}=$ independent component analysis. 

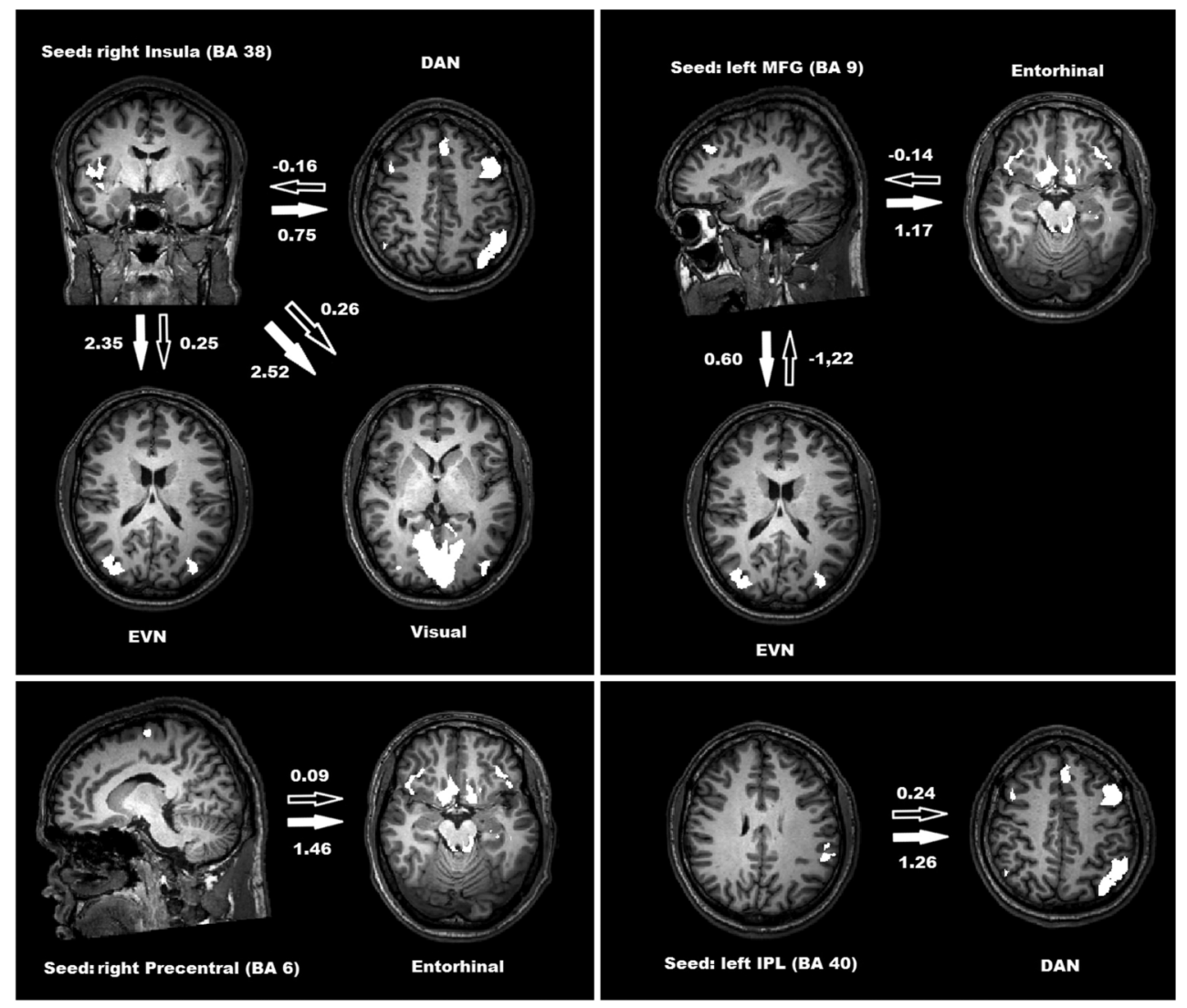

Figure 3. Significant Granger causality mapping (GCM) values from seed volume-of-interest to resting-state networks $(P<0.05$, Student $t$-test). Black arrows show GCM values in standard acquisition and white arrows show GCM values in silent echo-planar imaging acquisition. DAN = dorsal attention network; EVN = extrastriate visual network; MFG = middle frontal gyrus; Entorhinal = entorhinal network; IPL = inferior parietal lobule; BA = Brodmann areas.

in the hippocampus, indicating that the activity may be integrated into the gross aspect of the DMN or may be isolated. Interestingly, in the standard EPI condition, the hippocampal component is more extensive than in the presence of lower noise, suggesting interplay between these areas and depending on the acoustic noise.

The robustness of each network was confirmed by our results, as previously reported $(10,12)$. Direct comparisons between each individual network yield a cluster that is not large enough or only sparse voxels. Regarding the investigation of differences in functional connectivity between noise conditions, the five main RSNs had to be pooled before the statistical test.

The more specific comparison between conditions also identified differences in a variety of brain areas (Table 2). The inferior parietal lobule (BA 40) is related to the network we refer to as dorsal attentive and is dedicated to associative processing, integrating information from visual, auditory, and motor areas. Differences in functional connectivity in the insula point to autonomic aspects of brain function since the right insula is suggested to monitor visceral and emotional awareness (35). Taken together with differences in middle frontal and precentral areas (BA 6 and 9), this may indicate that lower acoustic noise permits the brain to work in a mode closer to a natural setting. These results agree with those reported by Peelle et al. (9) who detected a lower signal in the inferior parietal cortex for the quiet sequence. Since their subjects performed an auditory task during EPI acquisition and considering that lower noise required less effort to identify the sentences, task performance in the quiet mode demanded less processing from the associative area (BA 40). Thus, this area may be responsible for differences in effective connectivity to areas dedicated to attentional processing. On the other hand, higher noise induced an overload in the DMN, more specifically in the posterior cingulate cortex (PCC), as reported by Gaab et al. (10). These authors also suggested that passive processing of scanner noise provided enough externa stimulation to diminish introspective processes thought to be mediated by the DMN. Table 2 shows spatial differences between the two noise conditions by identifying 
local areas, but does not reveal how the functional connectivity is affected.

Figure 3 represents the local areas that reached significant differences in Granger causality with the identified RSNs depending on the noise condition. The first characteristic that draws attention to global aspects of effective connectivity is the absence of acoustic noise effect from seed regions to the DMN. In fact, previous results have shown that the organization of the DMN remains unchanged even when a task is executed (36). As cited by Gaab et al. (10), the effects of noise may occur in a nonlinear fashion, and these differences are related to nonstationarity due to ongoing changes depending on the external environment. The second point to be raised is the absence of an effect with a seed in the posterior cingulate cortex. Although the functional connectivity inside the posterior cingulate cortex differs according to noise level, as shown by group ICA values, differences in the effective connectivity are not confirmed with any specific network.

We found different values for GCM depending on the acoustic noise level between the inferior insula (BA 38) and attentive and visual networks. This aspect of global connectivity results is confirmed by significant differences found in GCM values from the left middle frontal cortex (BA 9) and the entorhinal network. Both frontal insula and entorhinal cortices are thought to be important for mental activity since they house von Economo spindle neurons, which are conspicuous in the human species and are implicated in the binding of complex mental activity, consciousness, and episodic memory (37). From this point of view, our results may have important implications in the study of healthy brain maturation (38) since these higherorder processes are much influenced during growth.

The calculation of effective connectivity depending on acoustic noise levels and areas with previously demonstrated differences in functional connectivity shows that in almost all cases the seed regions Granger-caused the activity in some functional networks, that is, the Granger causality values were positive and higher during silent EPI acquisition. The only negative value appeared under standard EPI when the right middle frontal gyrus was defined as seed. This result indicates that, under standard levels of noise, the associative visual network Grangercauses the activity in a region that is thought to be responsible for intelligence, mental activity, and theory of mind (39). Also, BOLD activity in the entorhinal network, formed by medial temporal lobe structures and bilateral supraorbital cortices, known to be important for declarative memory (40), shows higher values of Granger

\section{References}

1. Mason MF, Norton MI, Van Horn JD, Wegner DM, Grafton ST, Macrae CN. Wandering minds: the default network and stimulus-independent thought. Science 2007; 315: 393-395, doi: $10.1126 /$ science. 1131295 . causality under silent acquisition.

In summary, our study shows for the first time the advantages of using a sound-attenuated functional image acquisition in a continuous sampling resting-state paradigm. It shows that lower levels of acoustic noise promote stronger temporal coupling between RSNs implicated in cognitive functions (dorsal attention and entorhinal) and areas dedicated to reasoning (BA 9), visceral selfmonitoring (BA 13) and associative areas (BA 40).

As a byproduct, our results raise the question about the implications of applying low noise sequences to the everyday research as a way to permit the appreciation of the RSNs, which have a behavior closer to real resting conditions. From the clinical point of view, lower noise sequences may be of benefit for the acquisition of data from children and patients who show increased cortical responsiveness to auditory stimulation (8). Also, they obey the safety rules for those who work every day in an MRI facility (23). Ergonomic factors related to MRI operators may be increasingly discussed, as equipment with even quieter EPI sequences becomes commercially available.

Under lower noise conditions, the networks reach wider areas, leaving room for higher oscillatory activity in the motor/auditory cortex, supplementary motor network, and inside the frontal executive network, which favor the brain to work in a mode closer to a natural setting.

On the other hand, higher noise induced an overload in the DMN, more specifically in the posterior cingulate cortex. Although the functional connectivity in this area is different depending on the noise levels, differences in the effective connectivity are not confirmed with any specific network, indicating the robustness of the default network.

Applying low noise MR acquisitions to research may allow the detection of subtle differences of the RSNs, not to mention that the environment is closer to real resting conditions. As indicated by our results, high intensity of scanner background noise influences the process of stimulus-independent thought and mind wandering and has important implications for resting-state functional connectivity studies.

\section{Acknowledgments}

Research supported by FAPESP (grant \#2011/ 05934-6 to C. Rondinoni), partially supported by the CInAPCe Program FAPESP (\#2005/56447-7), and by the Provost's Office for Research of Universidade de São Paulo Programa de Incentivo à Pesquisa (\#2011.1.9333.1.3; NAPNA-USP).
2. Buckner RL, Andrews-Hanna JR, Schacter DL. The brain's default network: anatomy, function, and relevance to disease. Ann N Y Acad Sci 2008; 1124: 1-38, doi: 10.1196/annals.1440.011. 
3. Raichle ME, MacLeod AM, Snyder AZ, Powers WJ, Gusnard DA, Shulman GL. A default mode of brain function. Proc Natl Acad Sci U S A 2001; 98: 676-682, doi: 10.1073/ pnas.98.2.676.

4. Bandettini PA, Jesmanowicz A, Van Kylen J, Birn RM, Hyde JS. Functional MRI of brain activation induced by scanner acoustic noise. Magn Reson Med 1998; 39: 410-416, doi: 10.1002/mrm.1910390311.

5. Di Salle F, Formisano E, Seifritz E, Linden DE, Scheffler K, Saulino C, et al. Functional fields in human auditory cortex revealed by time-resolved $\mathrm{FMRI}$ without interference of EPI noise. Neuroimage 2001; 13: 328-338, doi: 10.1006/ nimg.2000.0683.

6. Amaro E Jr, Williams SC, Shergill SS, Fu CH, MacSweeney $\mathrm{M}$, Picchioni $\mathrm{MM}$, et al. Acoustic noise and functional magnetic resonance imaging: current strategies and future prospects. J Magn Reson Imaging 2002; 16: 497-510, doi: 10.1002/jmri.10186.

7. Moelker A, Pattynama PM. Acoustic noise concerns in functional magnetic resonance imaging. Hum Brain Mapp 2003; 20: 123-141, doi: 10.1002/hbm.10134.

8. Schmitter S, Diesch E, Amann M, Kroll A, Moayer M, Schad LR. Silent echo-planar imaging for auditory FMRI. MAGMA 2008; 21: 317-325, doi: 10.1007/s10334-008-0132-4

9. Peelle JE, Eason RJ, Schmitter S, Schwarzbauer C, Davis $\mathrm{MH}$. Evaluating an acoustically quiet EPI sequence for use in fMRI studies of speech and auditory processing. Neuroimage 2010; 52: 1410-1419, doi: 10.1016/j.neuroimage.2010.05.015.

10. Gaab N, Gabrieli JD, Glover GH. Resting in peace or noise: scanner background noise suppresses default-mode network. Hum Brain Mapp 2008; 29: 858-867, doi: 10.1002/ hbm. 20578.

11. Tomasi D, Caparelli EC, Chang L, Ernst T. fMRI-acoustic noise alters brain activation during working memory tasks. Neuroimage 2005; 27: 377-386, doi: 10.1016/j.neuroimage.2005.04.010.

12. Langers $D R$, van Dijk P. Robustness of intrinsic connectivity networks in the human brain to the presence of acoustic scanner noise. Neuroimage 2011; 55: 1617-1632, doi: 10.1016/j.neuroimage.2011.01.019.

13. de Zwart JA, van Gelderen P, Kellman P, Duyn JH. Reduction of gradient acoustic noise in MRI using SENSE-EPI. Neuroimage 2002; 16: 1151-1155, doi: 10.1006/nimg.2002.1119.

14. Hennel F, Girard F, Loenneker T. "Silent" MRI with soft gradient pulses. Magn Reson Med 1999; 42: 6-10, doi: 10.1002/ (SICl)1522-2594(199907)42:1<6::AID-MRM2>3.0.CO;2-D.

15. Li M, Lim TC, Lee JH. Simulation study on active noise control for a 4-T MRI scanner. Magn Reson Imaging 2008; 26: 393-400, doi: 10.1016/j.mri.2007.08.003.

16. McJury M, Stewart RW, Crawford D, Toma E. The use of active noise control (ANC) to reduce acoustic noise generated during MRI scanning: some initial results. Magn Reson Imaging 1997; 15: 319-322, doi: 10.1016/S0730725X(96)00337-2.

17. Edelstein WA, Hedeen RA, Mallozzi RP, El-Hamamsy SA, Ackermann RA, Havens TJ. Making MRI quieter. Magn Reson Imaging 2002; 20: 155-163, doi: 10.1016/S0730725X(02)00475-7.

18. Moelker A, Wielopolski PA, Pattynama PM. Relationship between magnetic field strength and magnetic-resonancerelated acoustic noise levels. MAGMA 2003; 16: 52-55, doi: 10.1007/s10334-003-0005-9.

19. Hattori $Y$, Fukatsu $H$, Ishigaki T. Measurement and evaluation of the acoustic noise of a 3 Tesla MR scanner. Nagoya J Med Sci 2007; 69: 23-28.

20. Hall DA, Haggard MP, Akeroyd MA, Palmer AR Summerfield AQ, Elliott MR, et al. "Sparse" temporal sampling in auditory fMRI. Hum Brain Mapp 1999; 7: 213223, doi: 10.1002/(SICI)1097-0193(1999)7:3<213::AIDHBM5>3.0.CO;2-N

21. Schwarzbauer C, Davis MH, Rodd JM, Johnsrude I. Interleaved silent steady state (ISSS) imaging: a new sparse imaging method applied to auditory fMRI. Neuroimage 2006; 29: 774-782, doi: 10.1016/j.neuroimage.2005.08.025.

22. McJury M, Shellock FG. Auditory noise associated with MR procedures: a review. J Magn Reson Imaging 2000; 12 : $37-$ 45, doi: 10.1002/1522-2586(200007)12:1<37::AIDJMRI5>3.0.CO;2-I.

23. Shellock FG, Crues JV. MR procedures: biologic effects, safety, and patient care. Radiology 2004; 232: 635-652, doi 10.1148/radiol.2323030830.

24. Formisano E, Esposito F, Di Salle F, Goebel R. Cortexbased independent component analysis of fMRI time series. Magn Reson Imaging 2004; 22: 1493-1504, doi: 10.1016/ j.mri.2004.10.020.

25. Esposito $\mathrm{F}$, Scarabino $\mathrm{T}$, Hyvarinen A, Himberg J, Formisano E, Comani S, et al. Independent component analysis of fMRI group studies by self-organizing clustering. Neuroimage 2005; 25: 193-205, doi: 10.1016/j.neuroimage.2004.10.042

26. McKeown MJ, Makeig S, Brown GG, Jung TP, Kindermann SS, Bell AJ, et al. Analysis of fMRI data by blind separation into independent spatial components. Hum Brain Mapp 1998; 6: 160-188, doi: 10.1002/(SICI)1097-0193(1998)6:3<160::AIDHBM5>3.0.CO;2-1.

27. Mantini D, Perrucci MG, Del Gratta C, Romani GL, Corbetta M. Electrophysiological signatures of resting state networks in the human brain. Proc Natl Acad Sci U S A 2007; 104 13170-13175, doi: 10.1073/pnas.0700668104.

28. Friston KJ. Functional and effective connectivity: a review. Brain Connect 2011; 1: 13-36, doi: 10.1089/brain.2011.0008.

29. Roebroeck A, Formisano E, Goebel R. Mapping directed influence over the brain using Granger causality and fMRI. Neuroimage 2005; 25: 230-242, doi: 10.1016/j.neuroimage.2004.11.017.

30. Rondinoni C, dos Santos AC, Salmon CEG. Effect of the scanner background noise on the resting brain networks detected by functional magnetic resonance imaging. Rev Bras Física Med 2011; 51: 93-98.

31. Goebel R, Esposito F, Formisano E. Analysis of functional image analysis contest (FIAC) data with brainvoyager QX: From single-subject to cortically aligned group general linear model analysis and self-organizing group independent component analysis. Hum Brain Mapp 2006; 27: 392-401, doi: 10.1002/hbm.20249.

32. De Martino F, Gentile F, Esposito F, Balsi M, Di Salle F, Goebel R, et al. Classification of fMRI independent components using IC-fingerprints and support vector machine classifiers. Neuroimage 2007; 34: 177-194, doi: 
10.1016/j.neuroimage.2006.08.041.

33. Tomasi DG, Ernst T. Echo planar imaging at 4 Tesla with minimum acoustic noise. J Magn Reson Imaging 2003; 18: 128-130, doi: 10.1002/jmri.10326.

34. De Curtis M, Pare D. The rhinal cortices: a wall of inhibition between the neocortex and the hippocampus. Prog Neurobiol 2004; 74: 101-110, doi: 10.1016/j.pneurobio.2004.08.005.

35. Craig AD. Human feelings: why are some more aware than others? Trends Cogn Sci 2004; 8: 239-241, doi: 10.1016/ j.tics.2004.04.004.

36. Greicius MD, Srivastava G, Reiss AL, Menon V. Defaultmode network activity distinguishes Alzheimer's disease from healthy aging: evidence from functional MRI. Proc Natl Acad Sci U S A 2004; 101: 4637-4642, doi: 10.1073/ pnas.0308627101.

37. Allman JM, Tetreault NA, Hakeem AY, Manaye KF,
Semendeferi K, Erwin JM, et al. The von Economo neurons in frontoinsular and anterior cingulate cortex in great apes and humans. Brain Struct Funct 2010; 214: 495-517, doi: 10.1007/s00429-010-0254-0.

38. Fair DA, Cohen AL, Dosenbach NU, Church JA, Miezin FM, Barch DM, et al. The maturing architecture of the brain's default network. Proc Natl Acad Sci U S A 2008; 105: 40284032, doi: 10.1073/pnas.0800376105.

39. Baron-Cohen S, Ring HA, Wheelwright S, Bullmore ET, Brammer MJ, Simmons A, et al. Social intelligence in the normal and autistic brain: an fMRI study. Eur $J$ Neurosci 1999; 11: 1891-1898, doi: 10.1046/j.1460-9568.1999. 00621.x.

40. Squire LR, Stark CE, Clark RE. The medial temporal lobe. Annu Rev Neurosci 2004; 27: 279-306, doi: 10.1146/ annurev.neuro.27.070203.144130. 